\title{
Treatment of chronic dry eye: focus on cyclosporine
}

\author{
George D Kymionis \\ Dimitrios I Bouzoukis \\ Vassilios F Diakonis \\ Charalambos Siganos
}

Department of Ophthalmology, Vardinoyannion Eye Institute of Crete, University of Crete, Greece
Correspondence: George D Kymionis Vardinoyannion Eye Institute of Crete, University Of Crete, Medical School Department of Ophthalmology,

7II I 0 Heraklion, Crete, Greece

Tel +302810371800

Fax +302810394653

Email kymionis@med.uoc.gr

\begin{abstract}
To review the current treatment of chronic dry eye syndrome, focusing on cyclosporine A (CsA), a systematic literature search was performed using PubMed databases in two steps. The first step was oriented to articles published for dry eye. The second step was focused on the use of CsA in dry eye. A manual literature search was also undertaken based on citations in the published articles. The knowledge on the pathogenesis of dry eye syndrome has changed dramatically during the last few years. Inflammation and the interruption of the inflammatory cascade seem to be the main focus of the ophthalmologic community in the treatment of dry eye, giving the anti-inflammatory therapy a new critical role. The infiltration of T-cells in the conjuctiva tissue and the presence of cytokines and proteasis in the tear fluid were the main reason introducing the use of immunomodulator agents such as corticosteroids, cyclosporine, and doxycicline in order to treat dry eye syndrome. CsA emulsion is approved by the FDA for the treatment of dry eye, while clinical trials of this agent have demonstrated efficacy and safety of CsA. CsA seems to be a promising treatment against dry eye disease. New agents focused on the inflammatory pathogenesis of this syndrome in combination with CsA may be the future in the quest of treating dry eye. More studies are needed to determine the efficacy, safety, timing, and relative cost/effect of CsA.
\end{abstract}

Keywords: dry eye, cyclosporine A, inflammation, immunomodulator agents

\section{Introduction}

\section{Epidemiology}

Dry eye is a frequent disease. Among dry eye patients over the age of 65 in the US, $25 \%$ reported using artificial tears on a frequent basis and $73 \%$ visited an eye care professional during the previous year for this condition (Shein et al 1997).

It is estimated that approximately 7.1 million people over the age of 40 in the US experience symptoms of ocular irritation due to dry eye syndrome (Pflugfelder 2004), while more than $6 \%$ of the population over the age of 40 and more than $15 \%$ of the population over the age of 65 suffer from dry eye (Bjerrum 1997; Schein et all 1997; McCarty et all 1998).

The estimated global sales of artificial tears exceeded US\$540 million annually in 2002 (Harmon and Murphy 2003), whereas the total annual healthcare cost of 1,000 dry eye syndrome sufferers managed by ophthalmologists ranged from US\$0.27 million (95\% CI: \$0.20; US\$0.38 million) in France to US\$1.10 million (95\% CI: US\$0.70; US $\$ 1.50$ million) in the UK. A large proportion of dry eye patients is either self-treated or managed by their general practitioner (Clegg et al 2006).

\section{Diagnosis of dry eye}

Clinically there is a plethora of irritation symptoms associated with dry eye such as ocular burning, stinging, scratchiness, soreness, photophobia, blurred vision and foreign body sensation. Tear film stability can be assessed with the fluorescein tear 
break-up time test, measuring the interval in seconds between a complete blink and the first appearing dry spot or discontinuity in the precorneal film. Aqueous tear production is measured more commonly with Shirmer test, calculating the length in millimeters that a folded filter paper strip placed in the lower lid wets during a 5-minute test period. There are two ways to perform this test: a) Shirmer test I is performed without topical anesthesia, which evaluates better the ability of the ocular gland to respond to ocular surface stimulation; b) Shirmer test II (or Basic Secretion test) which is performed after topical anesthesia, evaluating better the basal tear secretion. Meibonian gland disease is diagnosed by biomicroscopic recognition of pathological signs such as ductal orifice metaplasia, reduced expressibility of meibonian gland secretions, increased viscosity of the expressed secretion and dropout of glandular acini. Conjunctival goblet cell density and epithelial morphology can be directly evaluated by cytology. The most practical clinical method for assessing the severity of dry eye is the ocular surface dye staining. Fluorescein, rose Bengal and lissamine green are use as diagnostic dyes for evaluating the staining. Fluorescein staining occurs when the epithelial barrier is disrupted, due to the loss of the epithelial cells, is well tolerated by patients and evaluates better corneal staining. Rose Bengal and lissamine green stain the conjunctiva more brightly than the cornea. Rose Bengal stains devitalized epithelial cells or cells without protective mucus layer, but cause transient irritation after installation. Lissamine green dyes degenerated or dead cells and produces less irritation than rose Bengal (Plufelder 2006).

However, many times symptoms and signs are not always specific and are often underestimated by the patient or under diagnosed by ophthalmologist (Schein et al 1997; Afonso et al 1999; Lin et al 2003).

\section{Definitions and classifications of dry eye}

In 1995 the National Eye Institute workshops, defined dry eye as "a disorder of the tear film due to tear deficiency or excessive evaporation that causes damage to the interpalpebral ocular surface and is associated with symptoms of discomfort" (Lemp 1995). In the Definition and Classification Subcommittee of the international Dry Eye Workshop in 2007, a new contemporary definition of dry eye disease was reported, supported within a comprehensive classification framework. In accordance with the committee, dry eye was defined as a multifactorial disease of the tears and ocular surface that results in symptoms of discomfort, visual disturbance, and tears film instability with potential damage to the ocular surface. It is accompanied by increased osmolarity of the tear film and inflammation of the ocular surface. The committee recommended a three part classification system. The first part was etiopathogenic, illustrating the multiple causes of dry eye. In this group dry eye was divided in two principal categories: aqueous deficient (Sjogren or non-Sjogren related) and evaporative (intrinsic or extrinsic causes); the second was mechanistic, showing the way each cause may act through a common pathway (tear hyperosmolarity and tear film instability); the third part was based on the severity of the disease (four groups correlated to visual symptoms, conjuctival injection, conjunctival staining, corneal staining, corneal/tears signs, lid/meibonian glands, tear break-up time, and Shirmer test), providing a rational basis for therapy (DEW 2007).

In 2006 in a Delphi panel approach to treatment recommendations by 17 international specialists on dry eye syndrome, a new term was proposed for dry eye disease: dysfunctional tear syndrome (DTS). In our study the most commonly used diagnostic test reported by more than half of the panelists for evaluating probable dry eye were fluorescein staining (100\%), tear break up time (94\%), Shirmer test (71\%), and rose Bengal staining (65\%). Panelists agreed on three particular relevant symptoms and historical elements to be considered: ocular discomfort (itch, scratch, burn, foreign body sensation, and/or photophobia), tear substitute requirements, and visual disturbance. There was a consensus that most cases of DTS have an inflammatory basis that either triggers or maintains the inflammation. However, there was an agreement on the difficulty in clearly identifying inflammation in most patients and consequently the panel agreed to subclassify the disease as either DTS with clinically apparent inflammation or DTS without clinically evident inflammation (Behrens et al 2006).

\section{Pathogenesis}

The ocular surface and the lacrimal gland are considered, studied, and treated as an integrated functional unit interconnected by neural sensory/autonomic reflex arcs. Sensory afferent nerves, which enervate the ocular surface, traffic along the ophthalmic branch of the trigeminal nerve to the Pont area of the central nervous system. After received inputs from cortical areas (emotional central nervous centers), efferent nerves, consisting of parasympathetic fibers traveling in the facial nerve and of sympathetic fibers from the paraspinal sympathetic 
chain, lead to the main and the accessory lacrimal gland (Pflugfelder et all 2000).

Over recent years, inflammation has been shown to be the key in the pathogenesis of this syndrome, as it seems to be the cause and the consequence of dry eye. Regardless of the triggering factor, a vicious cycle of inflammation may be developed on the ocular surface in patients with dry eye. A glandular dysfunction creating tear deficiency or instability could irritate the surface of the eye and promote inflammation, which increases further the tear deficiency (Jones et all 1998). The results are antigen presentation and cytokine secretion by the epithelial cells of the lacrimal gland which promote the activation of the T-cells lymphocytes. Finally, the T-cells secrete pro-inflammatory cytokines, increasing further the level of inflammation (Meggs 1993; Mircheff et al 1998; Gao et al 1998). Consequently, the tears will contain cytokines. The ocular surface reacts, promoting inflammatory response which consists of inflammatory cell infiltration, epithelial activation, increased concentrations of cytokines and other inflammatory factors, and increased activity of matrix-degrading enzymes (Baudouin et al 1997; Tishler et al 1998; Afonso et al 1999; Pflugfelder 1999; Pflugfelder 2000; Sobrin et al 2000). This knowledge concerning the inflammation pathogenic mechanism in dry eye syndrome alters also the therapeutic approach against this syndrome which is now based on anti-inflammatory agents.

\section{Therapy}

The therapy of dry eye depends on its severity. Based on the most recent concept, the armamentarium used to control dry eye comprises a large range of therapeutic strategies.

Modification of the environmental conditions that increase tear evaporation (eg, low humidity), avoiding the use of systemic medications with anticholinergic side effects (eg, antihistamines), occlusion of the lacrimal canaliculi (punctal occlusion), stimulation of tears production (oral secretagogues pilocarpine), and minimization of corneal exposure (tarsorrhaphy, gas permeable contact lenses). Although these treatment options are very useful, all of them are considered to be symptomatic therapeutic approaches and not pathogenic.

\section{Artificial tears}

Artificial tears provide temporal improvement in eye irritation and blurred vision symptoms, visual contrast sensitivity, tear break up time, and ocular surface dye staining. (Gifford et al 2006; Ousler et al 2007). Artificial tears contain polymers such as cellulose esters, polyvinyl alcohol, and povidone, which determine their viscosity, shear properties, retention time, and adhesion to the ocular surface. Gels have longer retention times than artificial tear solutions (Bron et al 1998; Wilson et al 1998).

\section{Anti-inflammatory therapy}

Anti-inflammatory therapy is considered to be the first "causative therapeutic approach" in the treatment of dry eye, since its objective is to interrupt the inflammatory cascade. Topical corticosteroids, tetracyclines, and cyclosporine A (CsA) are the drugs used in the anti-inflammatory therapy of dry eye.

\section{Corticosteroids}

Topical corticosteroids can be used in order to decrease ocular surface inflammation inhibiting MMPs (matrix metalloproteinasis), inflammatory cytokines and adhesion molecule production. They demonstrate satisfactory results as pulse therapy. In a retrospective study which included Sjogren syndrome patients with keratoconjunctivitis sicca (KCS), the administration of $1 \%$ solution of methylprednisolone (3 times a day for 2 weeks) relieved symptoms in all patients (Marsh and Pflugfelder 1999). Corticosteroids should not be administered for long-term use owing to the side effects they can provoke (steroid response increasing of IOP, cataractogenesis). Corticosteroids with minimal potential to raise IOP (fluorometholone and loteprendol etabonate) could be considered a safer approach. A randomized, double-masked, placebo-controlled study of loteprednol etabonate and its vehicle was conducted on 64 patients with delayed tear clearance and KCS. After 2 and 4 weeks of treatment, there was no change in IOP in the corticosteroid-treated group. Patients with the most severe inflammatory signs at entry showed a significantly greater decrease in central corneal fluorescein staining scores while a significant decrease in inferior bulbar conjunctival hyperemia was demonstrated after 2 weeks (Plugfelder et al 2004).

\section{Tetracyclines}

Tetracyclines have also a variety of anti-inflammatory properties such as inhibition of MMPs and interleukine-I (IL-I) production (Amin et al 1996; Shlopov et al 1999). Orally administrated they decrease ocular surface symptoms in patients with ocular rosacea (Frucht-Pery et al 1993; Akpek et al 1997) and in patients with recurrent corneal epithelial erosions (Hope-Ross et al 1994). 
The best tolerated tetracycline is doxycycline, which is effective in doses of $20-50 \mathrm{mg}$ orally twice a day for a treatment up to 4 weeks (Plugfelder 2004).

\section{Cyclosporine A}

Today CsA seems to represent a very promising treatment against dry eye syndrome since it is the first agent focused on the pathogenesis of this disease. It can be used for long term without presentation of the adverse effects characterizing the other anti-inflammatory agents.

\section{Methodology}

A systematic literature review was performed using PubMed databases in two steps. The first step was oriented to articles published for dry eye. The second step was focused on the use of CsA in dry eye. The search strategy was not limited by year of publication. A manual literature search was also undertaken based on citations in the published articles.

\section{Results}

Cyclosporine A is a fungal-derived peptide that inhibits T-cell activation and consequently inhibits the inflammatory cytokine production (selective inhibition of IL-I). In addition, CsA inhibits apoptosis by blocking the opening of the mitochondrial permeability transition pore (MPTP) (Matsuda and Koyasu 2000) and by increasing the density of conjuctival goblet cells (Kunert et al 2002).

In the US, CsA is commercially distributed as Restasis ${ }^{\circledR}$. This ophthalmic emulsion $(0.05 \%$ cyclosporine [Allergan, Inc. Irvine, CA, USA]) is preservative free packaged in unit-dose vials. Restasis has been a prescription drug in the US since April 2003 when it was approved by the FDA for patients whose tear production is presumed to be suppressed due to ocular inflammation associated with KCS.

CsA was used for years routinely as an oral immunosuppressor for organ transplantation. Its action is non toxic and reversible after the treatment. In ophthalmology prior to dry eye treatment, CsA was used also for the treatment of severe posterior segment inflammations, when administered systemically (iv) or orally (Masuda et al 1989). Systemically CsA was also used for the treatment of peripheral ulcerative keratitis associated with Wegener's granulomatosis, in severe Graves's ophthalmopathy, and for the prevention of the recurrence of graft rejection after keratoplasty (Prummel et al 1989; Nussenblatt et al 1991; Georganas et al 1996; Reinhard et al 1997).

All these indications were the result of CsA's pharmacokinetics, since extraocular and intraocular tissues can be reached by this agent through the systemic pathway after oral administration. In fact, after oral daily administration of $5 \mathrm{mg} / \mathrm{kg}$ daily, the concentration of CsA was measured to be $25-75 \mu \mathrm{g} / \mathrm{mL}$ in human tears (BenEzra et al 1990); systemic administration may be accompanied by severe side effects such as nephrotoxity and hypertension (Mihatsch et al 1998). As a result topical ocular delivery was proposed as a good alternative. Despite its poor intraocular penetration, topical CsA has been successfully used in dry eye syndrome. Another pharmacokinetic limitation was that CsA could not be prepared in a formulation based on aqueous ophthalmic vehicles because of both its hydrophobicity and its low aqueous solubility. Therefore, the agent was dissolved in vegetable oils (Lallemand et al 2003).

The potential of CsA for treating dry eye syndrome and its clinical expression KCS was initially tested in 1989 in dogs affected with spontaneous canine KCS. Tear production was increased. CsA caused marked regression of chronic corneal neovascularization and granulation even in eyes in which lacrimation failed to improve. Additional benefits of topical cyclosporine were: reduced mucopurulent conjunctivitis, rapid healing of non-healing corneal ulcers, and reduced dependence on frequent topical treatments of KCS (Kaswan et al 1989). The experimental results were verified by other similar studies in dogs (Morgan and Abrams 1991; Olivero et al 1991; Izci et al 2002). Furthermore, experiments in a mutant mouse model used for primary Sjogren's syndrome has demonstrated the anti-inflammatory effect of both topical and orally administrated CsA on the lacrimal gland (Tsubota et al 1998), whereas other experiments in mice suggested that CsA accelerates tear secretion by releasing neurotransmitters from sensory nerve endings, which interact with the parasympathetic nerves (Yoshida et al 1999). Similar conclusions were also demonstrated in studies with rabbits (Toshida et al 1998).

The use of CsA delivered as ointment or oil suspension was then studied for treatment of humans KCS. Topical CsA $2 \%$ in olive oil was investigated for its possible immunoregulatory role on the dry eye state in patients with secondary Sjogren's syndrome. Thirty eyes of 15 patients were randomized to undergo treatment with topical cyclosporine in olive oil, while an other group of 15 patients (30 eyes) received a placebo (which was the sterile olive oil used as a vehicle for the cyclosporine). There was a significant increase in the break-up time and a significant decrease in rose Bengal staining score between the cyclosporine and control groups at the end of the 2-month study period (Gunduz and Ozdemir 1994). Laibovitz et al 
(1993) conducted a study in which patients with KCS underwent 6 weeks of treatment with either cyclosporine $1 \%$ ophthalmic ointment or placebo, followed by 6 weeks of the alternative treatment. Cyclosporine ointment was associated with initial mild to moderate redness, itching, and burning that returned to baseline levels within 1-2 weeks. Rose Bengal results and results of 4 subjective (patient diary) efficacy parameters favored cyclosporine: foreign body sensation, overall symptoms, hours of symptom control per day, and overall effectiveness. No systemic adverse events or laboratory abnormalities occurred. Subsequently, CsA for treating KCS in patients with dry eye was evaluated in large multi-center randomized double-masked FDA clinical trials. An FDA phase 2, randomized, multicenter, double masked, parallel-group, dose-response clinical trial compared 4 different dose of CsA (CsA ophthalmic emulsion $0.05 \%, 0.1 \%$, $0.2 \%, 0.4 \%$ ) administrated twice daily into both eyes of 129 patients and 33 patients treated with a vehicle for 12 weeks, followed by a 4-week post-treatment observation period. In a subset of 90 patients with moderate to severe KCS, CsA was found to decrease rose Bengal staining, superficial punctuate keratitis, and symptoms of ocular discomfort. Although there was no clear dose-response relationship, CsA $0.1 \%$ seemed to produce the most consistent improvements in subjective and objective end points, whereas CsA $0.05 \%$ demonstrated the most consistent improvement in patients' symptoms. All treatments were well tolerated by patients (Stevenson et al 2000).

In the FDA phase 3 study group, 2 independent multicenter, randomized, double-masked, parallel group, 6-month, vehicle control clinical trials compared the efficacy and safety of CsA in 877 patients with defined moderate to severe dry eye disease. The patients were treated twice daily with either CsA $0.05 \%$ or $0.1 \%$, or vehicle ( 292 to 293 patients in each group). The results of these two trials were combined for analysis. There was no dose-response effect. A treatment effect was observed after first month of treatment and increased over the 6-month follow-up period. Patients treated with CsA $(0.05 \%$ or $0.1 \%$ ) showed significantly greater improvements than vehicle in 2 objective signs (corneal fluorescein staining and categorized Shirmer values). CsA $0.05 \%$ treatment also demonstrated significantly greater improvements in three subjective measures of dry eye (blurred vision, need for concomitant artificial tears, and physician's evaluation of global response to treatment). After 6 months of treatment there was a statistically significant improvement from baseline within both groups in Shirmer tear test obtained with anesthesia, whereas in those obtained without anesthesia there was a statistically significant improvement from baselines within all treatment groups but no statistically significant differences among the groups. The most common treatment-related adverse effect was burning eye sensation (15\% for CsA $0.05 \%$ group and $17 \%$ for CsA $0.1 \%$ group). Patients receiving the vehicle had a lower frequency of burning eye sensation (7\%). Other common adverse events (reported by $>3 \%$ of patients) were stinging eye, eye discharge, foreign body sensation, conjunctival hyperemia, visual disturbance, and eye pain (Sall et al 2000).

A multicentered, non-randomized, open label extension study of the two previous phase 3 clinical trials, evaluated the safety and efficacy of CsA $0.1 \%$ ophthalmic emulsion over 1-3 years in moderate to severe dry eye disease patients. In 412 patients previously dosed for 6-12 months with CsA $0.05 \%$ or $0.1 \%$, CsA $0.1 \%$ was instilled twice daily for up to 3 consecutive 12-month periods. For the duration of almost all the 20-month treatment, improvements in objective and subjective measures of dry eye were modest. The changes in corneal staining and Shirmer scores were not statistically significant. Treatment-related adverse effects were found in $22 \%$ of patients. The most common were burning eye, stinging eye, and conjunctival hyperemia, but only 2 patients reported these symptoms as severe. In a survey of patients receiving CsA during a 12-month treatment period, most patients reported that during the first clinical trial preceding this study, their symptoms began to resolve after the first 3 months. Although $85 \%$ of respondents continued to use artificial tears, their frequency decreased from 7.6 times per day to 3.8 times per day. $95.2 \%$ of respondents said that they would continue taking CsA after the study and $98 \%$ would recommend it to other patients. A 2 line loss of visual acuity was found in $13 \%$ of patients, whereas only $5 \%$ showed a gain of 2 lines (Barber et al 2005).

The use of CsA for the treatment of dry eye was also tested in a variety of situations correlated with this syndrome. It is well known that LASIK, despite its positive attributes in keratorefractive surgery, can induce dry eye syndrome in some patients (Aras et al 2000; Battat et al 2001; Toda et al 2001; Nassaralla et al 2003). Dry eye in patients that underwent LASIK seems to be a result of multifactorial processes such as decreased corneal sensation or alteration ocular surface and lacrimal gland function (Heigle and Plugfelder 1996; Linna et al 2000; Stern et al 2000). As a result, dry eye is considered to be almost a contraindication for LASIK surgery. A prospective clinical trial evaluated the safety and efficacy of CsA $0.05 \%$ versus artificial tears in dry eye patients, from 1 month before to 3 months after 
having LASIK. There were no statistically significant differences between baselines or between the two groups in response to ocular surface disease index questionnaire or best spectacle-corrected visual acuity. Treatment with CsA provided greater refractive predictability than artificial tears 3 and 6 months after surgery (Salib et al 2006).

Rao and Rao (2006) assessed the efficacy of cyclosporine in patients with dry eye associated with draft versus host disease after stem cells transplantation. After completing a 3-month run-in period of using only artificial tears to control dry eye symptoms in both eyes, patients who failed to achieve adequate relief were instructed to instill topical cyclosporine twice a day. Dry eye signs improved significantly with cyclosporine treatment.

Based on its anti-inflammatory action, topical CsA could also be used in a variety of other ocular surface disease such as vernal keratoconjutivitis (Pucci et al 2002), atopic keratoconjutivitis (Akpek et al 2004), ligneous conjutivitis (Rubin et al 1991), conjuctival linchen planus (Levell et al 1992), and superior limbic keratoconjutivitis (Perry et al 2003).

The concomitant use of cyclosporine with artificial tears has also been analyzed. Sal et al (2006) evaluated the efficacy of marked artificial tears in relieving the signs and symptoms of dry eye when used as a supportive therapy to a cyclosporinebased ophthalmic emulsion in a 6-month randomized investigator masked parallel study of 61 patients. The patients were divided in 3 groups; in the first two cyclosporine was combined with a preservative-free carboxymethylcellulose $0.5 \%$ agent (Refresh Plus ${ }^{\circledR}$, Allergan, Irvine, CA, USA) and with a hydroxypropyl-guar gellable lubricant eye drop (Systane ${ }^{\circledR}$, Alcon laboratories, Inc., Fort Worth, TX, USA), whereas in the third group only Systane was used. Results indicated that the choice of concomitant therapy had significant effects on outcome measures. The combination of Restasis-Systane was statistically significantly better in reducing the signs and symptoms of dry eye than the combination of RestasisRefresh. In addition, Systane used alone was statistically significantly better than Restasis-Refresh for 3 ocular symptom frequency scales (burning, dryness, and scratchiness) and 6 Likert acceptability questions. Finally, Restasis-Systane, compared with Systane alone, was not statistically different for any of the signs and symptoms measured except for the frequency of ocular blurring (Sal et al 2006).

\section{Discussion}

Although traditional symptomatic treatment still remains the method of choice, a more reasonable approach in the treatment of this syndrome would seem to be consider the inflammatory mechanism of dry eye. The trend nowadays in patients with slight to mild episodic dry eye seems to be symptomatic therapy (eg, artificial tears, punctual occlusion), while in patients who already use artificial tears and who continue to have detectable ocular surface disease associated with inflammatory signs, anti-inflammatory agents seem to be the treatment of choice.

The use of CsA as anti-inflammatory treatment for dry eye has advantages over corticosteroids because until now a) it has not demonstrated serious adverse effects; $b$ ) its action is reversible after the treatment; and c) it has a very low rate of systemic absorption. These pharmacokinetic characteristics are critical, since the possibility for long-term therapy in these patients is absolutely necessary for a chronic disease such as dry eye. In fact, the benefits of CsA begin after 1 moth of treatment and a therapy of at least 3 months seems to be indispensable.

Also other conditions associated with dry eye could benefit from CsA. For example, refractive surgery of the cornea, most particularly LASIK, is almost contraindicated now in patients with dry eye. CsA treatment before and after surgery could help these patients obtain a surgical correction of their refractive error without the risk of dry eye. Graft versus Host disease cells after stem transplantation could also benefit from CsA. Similar results were be found in other pathologies with an inflammatory base, such as vernal kerato-conjunctivitis, atopic kerato-conjunctivitis, ligneous conjunctivitis, and superior limbic kerato-conjunctivitis.

Plugfelder (2004) reviewed anti inflammatory therapy for chronic dry eye. He stated that dry eye is associated with ocular surface inflammation that may further compromise tear secretion and cause ocular surface disease and irritation symptoms. Ocular surface inflammation should be assumed in patients with an unstable tear film and ocular surface epithelial disease that is detected by staining with diagnostic dyes. Of course, there is no diagnostic test available to gauge easily the level of inflammation on the ocular surface. As a result, no firm recommendations can be made about the optimal timing for initiating anti-inflammatory therapy in patients with dry eye. He concluded that it is reasonable to consider anti-inflammatory therapy for patients using artificial tears who continue to have clinically detectable ocular surface disease, particularly if inflammatory signs (for example, conjunctival redness) and irritation symptoms are present.

The Delphi panel in 2006 proposed treatment recommendation for dry eye on the basis of level of severity. They recommended 
that topical administration of CsA must be given only when the disease is in severity level two, characterized by moderate to severe symptoms, tear film signs, mild cornea punctuate staining, conjunctival staining, and visual signs.

However, a new treatment must take consider the relationship between cost and effect. Miljanovic et al (2007) measured the impact of dry eye syndrome on vision-related quality of life. They asked 190 participants with clinically diagnosed dry eye or severe symptoms and 399 without dry eye to complete a questionnaire, asking how much their everyday activities were limited by symptoms of dry eye and to what degree problems with their eyes limited their reading, driving, working at the computer, professional activities, and watching television. They concluded that dry eye syndrome is associated with a measurable adverse impact on several common and important tasks of daily living, further implicating this condition as an important public health problem deserving increased attention and resources. Clegg et al (2006) estimated the annual cost associated with the management of dry eye patients by ophthalmologists in France, Germany, Italy, Spain, Sweden, and the UK from the perspective of the health-care systems in the respective countries. They concluded that dry eye syndrome did not appear to impose a direct burden on the health care expenditure of the country investigated (Clegg et al 2006). However, among these countries CsA was used only in Germany for severe cases associated with Sjogren's syndrome. CsA costs much more than other medicines and the economic impact of this medicine must be seriously considered in relation to its effect on dry eye, given that it not only substitutes for but also complements the existing treatments for dry eye.

It is likely that future clinical and epidemiological studies will provide more definitive recommendations about the timing, efficacy, safety, and relative costs/benefits of anti-inflammatory therapy with CsA for dry eye.

\section{Disclosures}

The authors have no financial or proprietary interest in any materials or methods described herein.

\section{References}

Afonso A, Monroy D, Stern M, et al. 1999. Correlation of tear fluorescein clearance and Schirmer test scores with ocular irritation symptoms. Ophthalmology, 106:803-10.

Afonso A, Sobrin L, Monroy D, et al. 1999. Tear fluid pro-gelatinase B activity correlates with tear clearance and IL-1 concentration in patients with ocular rosacea. Invest Ophthalmol Vis Sci, 40:2506-12.

Akpek EK, Dart JK, Watson S, et al. 2004. A randomized trial of topical cyclosporine $0.05 \%$ in topical steroid-resistant atopic keratoconjunctivitis. Ophthalmology, 111:476-82.
Akpek EK, Merchant A, Pinar V, et al. 1997. Ocular rosacea: patient characteristics and follow-up. Ophthalmology, 104:1863-7.

Amin AR, Attur MG, Thakker GD, et al. 1996. A novel mechanism of action of tetracyclines: effects on nitric oxide synthases. Proc Natl Acad Sci USA, 93:14014-19.

Aras C, Ozdamar A, Bahcecioglu H, et al. 2000. Decreased tear secretion after laser in situ keratomileusis for high myopia. J Refract Surg, $16: 362-4$.

Barber L, Pflugfelder S, Tauber J et al. 2005. Phase III safety evaluation of cyclosporine $0.1 \%$ ophthalmic emulsion administered twice daily to dry eye disease patients for up to 3 years. Ophthalmology, 112:1790-4.

Battat L, Macri A, Dursun D, et al. 2001. Effects of laser in situ keratomileusis on tear production, clearance, and the ocular surface. Ophthalmology, 108:1230-5.

Baudouin C, Brignole F, Becquet F, et al. 1997. Flow cytometry in impression cytology specimens. Invest Ophthalmol Vis Sci, 38:1458-64.

Behrens A, Doyle JJ, Stern L, et al. 2006. Dysfuncional tear syndrome. A Delphi approach to treatment recommendations. Cornea, 25:900-7.

BenEzra D, Maftzir G, de Courten C, et al. 1990. Ocular penetration of cyclosporine A. III: The human eye. Br J Ophthalmol, 74:350-2.

Bjerrum K. 1997. Keratoconjunctivitis sicca and primary Sjogren's syndrome in a Danish population aged 30-60 years. Acta Ophthalmol Scand, 75:281-6.

Bron A, Daubas P, Siou-Mermet R, et al. 1998. Comparison of the efficacy and safety of two eye gels in the treatment of dry eyes: Lacrinorm and Viscotears. Eye, 12:839-47.

Georganas C, Ioakimidis D, Iatrou C, et al. 1996. Relapsing Wegener's granulomatosis: successful treatment with cyclosporin-A. Clin Rheumatol, 15:189-92.

Clegg J, Guest J Lehman A, et al. 2006. The annual cost of dry eye syndrome in France, Germany, Italy, Spain, Sweden and the United Kingdom among patients managed by ophthalmologists. Ophthalmic Epidemol, 13:263-74.

DEW. 2007. The definition and classification of dry eye disease: report of the Definition and Classification Subcommittee of the International Dry Eye Workshop (2007) Ocul Surf, 5:75-92.

Frucht-Pery J, Sagi E, Hemo I, et al. 1993. Efficacy of doxycycline and tetracycline in ocular rosacea. Am J Ophthalmol, 116:88-92.

Gao J, Schwalb TA, Addeo JV, et al. 1998. The role of apoptosis in the pathogenesis of canine keratoconjunctivitis sicca: the effect of topical Cyclosporin A therapy. Cornea, 17:654-63.

Gifford P, Evans B, Morris J. 2006. A clinical evaluation of Systane. Cont Lens Anterior Eye, 29:31-42.

Gunduz K, Ozdemir O. 1994. Topical cyclosporin treatment of keratoconjunctivitis sicca in secondary Sjogren's syndrome. Acta Ophthalmol (Copenh), 72:438-42.

Harmon D, Murphy R. 2003. Dry eye market set for robust growth. Market Scope, 8:7-8.

Heigle TJ, Pflugfelder SC. 1996. Aqueous tear production in patients with neurotrophic keratitis. Cornea, 15:135-8.

Izci C, Celik I, Alkan F, et al. 2002. Histologic characteristics and local cellular immunity of the gland of the third eyelid after topical ophthalmic administration of $2 \%$ cyclosporine for treatment of dogs with keratoconjunctivitis sicca. Am J Vet Res, 63:688-94.

Jones DT, Ji A, Monroy D, et al. 1998. Evaluation of ocular surface cytokine, mucin, and cytokeratin expression in Sjogren's Syndrome. Adv Exp Med Biol, 438:533-6.

Masuda K, Nakajima A, Urayama A, et al. 1989. Double-masked trial of cyclosporin versus colchicine and longterm open study of cyclosporin in Behcet's disease. Lancet, 1:1093-6.

Kaswan R, Salisbury M, Ward D. 1989. Spontaneous canine keratoconjunctivitis sicca. A useful model for human keratoconjunctivitis sicca: treatment with cyclosporine eye drops. Arch Ophthalmol, 107:1210-16.

Kunert KS, Tisdale AS, Gipson IK. 2002. Goblet cell numbers and epithelial proliferation in the conjunctiva of patients with dry eye syndrome treated with cyclosporine. Arch Ophthalmol, 120:330-7. 
Laibovitz RA, Solch S, Andrianao J. 1993. Pilot trial of cyclosporine 1\% ophthalmic ointment in the treatment of keratoconjunctivitis sicca. Cornea, 12:315-23.

Lallemand F, Felt-Baeyens O, Besseghir Ket al. 2003. Cyclosporine A delivery to the eye: A pharmaceutical challenge. Eur J Pharm Biopharm, 56:307-18.

Lemp MA. 1995. Report of the National Eye Institute/Industry Workshop on Clinical Trials in Dry Eyes. CLAO J, 21:222-32.

Levell NJ, Munro CS, Marks JM. 1992. Severe lichen planus clears with very low dose cyclosporin. Br J Dermatol, 127:66-7.

Lin P, Tsai S, Cheng C, et al. 2003. Prevalence of dry eye among an elderly Chinese population in Taiwan: the Shihpai Eye Study. Ophthalmology, 110:1096-101.

Linna TU, Vesaluoma MH, Perez-Santonja JJ, et al. 2000. Effect of myopic LASIK on corneal sensitivity and morphology of subbasal nerves. Invest Ophthalmol Vis Sci, 41:393-7.

Marsh P, Pflugfelder SC. 1999. Topical non-preserved methylprednisolone therapy of keratoconjunctivitis sicca in Sjogren's syndrome. Ophthalmology, 106:811-6.

McCarty C, Bansal A, Livingston P, et al. 1998. The epidemiology of dry eye in Melbourne, Australia. Ophthalmology, 105:1114-9.

Meggs WJ. 1993. Neurogenic inflammation and sensitivity to environmental chemicals. Environ Health Perspect, 101:234-8.

Mihatsch M, Kyo M, Morozumi K, et al. 1998. The side-effects of ciclosporine-A and tacrolimus. Clin Nephrol, 49:356-63.

Miljatovic B, Dana R, Sullivan D et al. 2007. Impact of dry eye syndrome on vision-related quality of life. Am J Ophthalmol, 143:409-15.

Mircheff A, Gierow JP, Yang T, et al. 1998. Sjogren's autoimmunity: how perturbation of recognition in endomembrane traffic may provoke pathological recognition at the cell surface. J Mol Recognit, $11: 40-8$.

Morgan RV, Abrams KL. 1991. Topical administration of cyclosporine for treatment of keratoconjunctivitis sicca in dogs. J Am Vet Med Assoc, 199:1043-6.

Nassaralla BA, McLeod SD, Nassarala JJ Jr. 2003. Effect of myopic LASIK on human corneal sensitivity. Ophthalmology, 110:497-502.

Nussenblatt R, Palestine A,Chan C, et al. 1991. Randomized, double-masked study of cyclosporine compared to prednisolone in the treatment of endogenous uveitis. Am J Ophthalmol, 112:138-46.

Olivero DK, Davidson MG, English RV, et al. 1991. Clinical evaluation of $1 \%$ cyclosporine for topical treatment of keratoconjunctivitis sicca in dogs. J Am Vet Med Assoc, 199:1039-42

Ousler G, Michaelson C, Christensen M. 2007. An evaluation of tear film break-up time extension and ocular protection index scores among three marketed lubricant eye drops. Cornea, 26:949-52.

Perry HD, Doshi-Carnevale S, Donnenfeld ED, et al. 2003. Topical cyclosporin A $0.5 \%$ as a possible new treatment for superior limbic keratoconjunctivitis. Ophthalmology, 110:1578-81.

Pflugfelder S, Jones DT, Ji Z, et al. 1999. Altered cytokine balance in the tear fluid and conjunctiva of patients with Sjogren's syndrome keratoconjunctivitis sicca. Curr Eye Res, 19:201-11.

Pflugfelder S, Solomon A, Stern M. 2000. The diagnosis and management of dry eye. a twenty-five-year review. Cornea, 19:644-9.

Pflugfelder SC Maskin SL, Anderson B et al. 2004. A randomized, double-masked, placebo-controlled, multicenter comparison of loteprednol etabonate ophthalmic suspension, $0.5 \%$, and placebo for treatment of keratoconjunctivitis sicca in patients with delayed tear clearance. Am J Ophthalmol, 138:444-57.

Pflugfelder SC. 2004. Antiinflammatory therapy for dry eye. Am J Ophthalmol, 137:337-42.

Plugfelder SC. 2006. Dry eye. Focal Points (AAO), 14(5).
Prummel M, Mourits M, Berghout A, et al. 1989. Prednisone and cyclosporine in the treatment of severe Graves' ophthalmopathy. $N$ Engl J Med, 321:1353-9.

Pucci N, Novembre E, Cianferoni A, et al. 2002. Efficacy and safety of cyclosporine eyedrops in vernal keratoconjunctivitis. Ann Allergy Asthma Immunol, 89:298-303.

Rao S, Rao R. 2006. Efficacy of topical cyclosporine $0.05 \%$ in the treatment of dry eye associated with graft versus host disease. Cornea, 25:674-8.

Reinhard T, Sundmacher R, Godehardt E, et al. 1997. Preventive systemic cyclosporin A after keratoplasty at increased risk for immune reactions as the only elevated risk factor. Ophthalmologe, 94:496-500.

Rubin BI, Holland EJ, de Smet MD, et al. 1991. Response of reactivated ligneous conjunctivitis to topical cyclosporin. Am J Ophthalmol, 112:95-6.

Salib G, McDonald M, Smolek M. 2006. Safety and efficacy of cyclosporine $0.05 \%$ drops versus unpreserved artificial tears in dry-eye patients having laser in situ keratomileusis. J Cataract Refract Surg, 32:772-8.

Sall K, Cohen S, Christensen M. 2006. An evaluation of the efficacy of a Cyclosporine-based dry eye therapy when used with marked artificial tears as a supportive therapy in dry eye. Eye Contact Lens, $32: 21-6$.

Sall K, Stevenson OD, Mundorf TK, et al. 2000. Two multicenter, randomized studies of the efficacy and safety of cyclosporine ophthalmic emulsion in moderate to severe dry eye disease. Ophthalmology, 107:631-9.

Schein O, Munoz B, Tielsch J, et al. 1997. Prevalence of dry eye among the elderly. Am J Ophthalmology, 124:723-8.

Schein O, Munoz B, Tielsch J, et al. 1997. Prevalence of dry eye among the elderly. Am J Ophthalmol, 124:723-8.

Schein O, Tielsch J, Munoz B, et al. 1997. Relation between signs and symptoms of dry eye in the elderly: a population-based perspective. Ophthalmology, 104:1395-401.

Shlopov BV, Smith GN, Cole AA et al. 1999. Differential patterns of response to doxycycline and transforming growth factor beta1 in the down-regulation of collagenases in osteoarthritic and normal human chondrocytes. Arthritis Rheum, 42:719-27.

Sobrin L, Liu Z, Monroy D, et al. 2000. Activators and inhibitors of matrix metalloproteinase-9 in human tear fluid and corneal epithelial culture supernatant. Invest Ophthalmol Vis Sci, 41:1703-9.

Stern ME, Beuerman RW, Fox RIet al. 1998. The pathology of dry eye: the interaction between the ocular surface and lacrimal glands. Cornea, 17:584-9.

Stevenson D, Tauber J, Reis BL. 2000. Efficacy and safety of cyclosporin A ophthalmic emulsion in the treatment of moderate-to-severe dry eye disease a dose-ranging, randomized trial. Ophthalmology, 107:967-74.

Tishler M, Yaron I, Geyer D, et al.1998. Elevated tear interleukin-6 levels in patients with Sjogren's syndrome. Ophthalmology, 105:2327-9.

Toda I, Asano-Kato N, Komai-Hori Y, et al. 2001. Dry eye after laser in situ keratomileusis. Am J Ophthalmol, 132:1-7.

Toshida H, Nakayasu K, Kanai A. 1998. Effect of Cyclosporin A Eyedrops on Tear Secretion in Rabbit. Jpn J Ophthalmol, 42:168-73.

Tsubota K, Saito I, Ishimaru N et al. 1998.Use of topical cyclosporin A in a primary Sjogren's syndrome mouse model. Invest Ophthalmol Vis Sci, 39:1551-9.

Wilson C, Zhu Y, Fier M et al. 1998. Ocular contact time of a carbomer gel (GelTears) in humans. Br J Ophthalmol, 82:1131-4.

Yoshida A, Fujihara T, Nakata K. 1999. Cyclosporine increase tear fluid secretion via release of sensory neurotransmitters and muscarinic pathway in mice. Exp Eye Res, 68:541-6. 